\title{
Wear and Corrosion Resistance of CoCrFeNiSiMoW Medium-Entropy Alloy Coatings on Q235 Steel
}

\author{
Qingxian $\mathrm{Hu}^{*}$, Xiaoli Wang *, Xinwang Shen, Fanglian Fu and Zemin Tan \\ School of Materials Science and Engineering, Jiangsu University of Science and Technology, \\ Zhenjiang 212003, China; 1440602222@stu.just.edu.cn (X.S.); flfu1997@stu.just.edu.cn (F.F.); \\ 199060046@stu.just.edu.cn (Z.T.) \\ * Correspondence: huqingxian@just.edu.cn (Q.H.); xlwang@just.edu.cn (X.W.)
}

check for updates

Citation: Hu, Q.; Wang, X.; Shen, X.; Fu, F.; Tan, Z. Wear and Corrosion Resistance of CoCrFeNiSiMoW Medium-Entropy Alloy Coatings on Q235 Steel. Coatings 2021, 11, 1053. https://doi.org/10.3390/ coatings11091053

Academic Editors: Ludmila

B. Boinovich and

Diego Martinez-Martinez

Received: 7 July 2021

Accepted: 28 August 2021

Published: 31 August 2021

Publisher's Note: MDPI stays neutral with regard to jurisdictional claims in published maps and institutional affiliations.

Copyright: (c) 2021 by the authors. Licensee MDPI, Basel, Switzerland. This article is an open access article distributed under the terms and conditions of the Creative Commons Attribution (CC BY) license (https:/ / creativecommons.org/licenses/by/ $4.0 /)$.

\begin{abstract}
CoCrFeNiSiMoW medium-entropy alloy coatings (MEACs) were fabricated by plasma-arc surfacing welding on Q235 steel. The microstructures and mechanical properties of CoCrFeNiSiMoW MEACs were studied. CoCrFeNiSiMoW MEACs are made from a mixture of NiCrCoMo cubic (FCC) solid solution phase, (Fe, Ni), Mo1.24Ni0.76, and CoCx phases by XRD analysis. The average hardness values of the one- and two-layer CoCrFeNiSiMoW MEACs obtained were $186 \pm 1.56$ and $198 \pm 1.78 \mathrm{HV}$, respectively. Compared with the one-layer CoCrFeNiSiMoW coating, the two-layer coating has a better wear performance due to its higher hardness. Its corrosion resistance is better because of its higher Ni content.
\end{abstract}

Keywords: CoCrFeNiSiMoW; medium-entropy alloy coatings; microstructure; wear

\section{Introduction}

Every year, the economic losses caused by the wear and corrosion of metal materials are high. At the same time, the wear and corrosion of metal materials also affect the quality of industrial products, which contribute to a poor market image for enterprises, and will inevitably bring challenges to the realization of economic benefits for enterprises. It is particularly important to prepare wear- and corrosion-resistant coatings on the surface of the material. Due to the increasing demand of modern industry for wear- and corrosionresistant coatings, researchers continue to explore new coating materials.

High- and medium-entropy alloys (HEAs and MEAs, respectively) possess a list of excellent properties, such as excellent strength and hardness [1,2], outstanding wear performance [3], and good corrosion resistance [4,5]. When HEA and MEA were initially proposed, they were considered to be composed of at least five elements. After an in-depth study of HEAs and MEAs, some quaternary alloys not equal to or close to equal atomic percentages, ranging from $5 \%$ to $35 \%$, were also determined to be considered HEAs and MEAs [6]. It provided the new idea to fabricate alloy coatings with good wear and corrosion resistance due to their high content of multiple corrosion-resistant elements [7]. Many types of research about high- and medium-entropy alloy coatings (HEACs and MEACs, respectively) have been conducted. Feng et al. [8] researched the corrosion properties of laser cladding CrCoNi MEACs and found they had better corrosion resistance than SS304. Zhao et al. [9] studied the fabrication and friction properties of an AlFeCrCo MEAC on a magnesium alloy using a resistance seam-welding method. The results showed that the AlFeCrCo MEAC has a better wear performance than the substrate. Tian et al. [10] synthesized a CrMnFeCoNi HEAC with a thickness of $180 \mu \mathrm{m}$ on Q235 steel using a mechanical alloying method and found it had better corrosion resistance than Q235 steel in a $3.5 \% \mathrm{NaCl}$ solution. A Al2CrFeNiMox HEAC was fabricated by laser cladding, and its wear resistance was improved because of the properties of Mo [11]. Xu et al. [12] fabricated a CoCrFeNiTiMo HEAC and studied its corrosion resistance. Lloyd et al. [13] studied the effects of the elements $\mathrm{Cr}$, Mo, and $\mathrm{W}$, on the passive film of Ni-Cr-Mo (W) alloys in acidic 
solutions. Their results showed that high Cr-alloys form thicker oxides with a core-shell structure consisting of an inner $\mathrm{Cr}-\mathrm{Ni}$ oxide layer and an outer $\mathrm{Mo} / \mathrm{W}$ oxide in a $0.1 \mathrm{M}$ $\mathrm{H}_{2} \mathrm{SO}_{4}+1.0 \mathrm{M} \mathrm{NaCl}$ solution. Some studies have shown that Mo can also alter mechanical properties and the corrosion resistance of HEAs because it can change the morphology, the segregation behavior of elements, and the phase structure [14,15]. In addition, $\mathrm{Cr}$ can improve the wear and corrosion resistance of materials, and $\mathrm{Ni}$ can help improve the corrosion performance in acidic solutions.

The methods of fabricating HEACs include laser cladding [16], magnetron sputtering [17], plasma spraying [18], and more. Laser cladding technology is a common technique to fabricate wear-resistant coatings. In the process of laser cladding, the heat source is a laser, and the cladding material is a powder material. The cladding efficiency is relatively low and the price of power is high. Compared to laser cladding, plasma-arc surfacing technology uses welding wire as a cladding material and a plasma arc as a heat source, which has a relatively higher efficiency and is a more economical preparation method of cladding coating. In this process, the dilution effect of the base metal affects the mechanical properties of coatings. The Fe element from the base metal can change the proportion of elements in coatings so that the HEA/MEA coatings can be obtained by making full use of the dilution and selecting the appropriate welding wire. To study the effects of this change on the microstructure and properties, one- and two-layer coatings were prepared.

Hastelloy X (HX) alloy contains $\mathrm{Cr}, \mathrm{Ni}$, and $\mathrm{Mo}$, and it was selected to fabricate HEACs/MEACs by plasma-arc surfacing welding in this study. HX has been widely studied in high temperature conditions because it has extraordinary properties, such as formability, oxidation resistance, and mechanical properties in the temperature range of 540-1000 ${ }^{\circ} \mathrm{C}$ [19-21]. However, the literature on fabricating HEACs/MEACs with HX and about its wear and corrosion resistance at room temperature is plentiful.

In this work, one- and two-layer CoCrFeNiSiMoW MEACs were fabricated with HX wire by plasma-arc surfacing welding, and its wear and corrosion resistance were studied.

\section{Experimental Details}

\subsection{CoCrFeNiSiMoW Coating Fabrication}

Q235 steel was taken as the base metal in this study. Before plasma-arc surfacing welding, the surface of Q235 was ground with 200\# sandpaper and cleaned with acetone. The HX wire was used as filling wire to fabricate the coatings. The main elemental compositions of Q235 and HX wire are shown in Table 1.

Table 1. The main elemental compositions of Q235 steel and HX wire (wt.\%).

\begin{tabular}{ccccccccc}
\hline Elements & Mn & Si & W & Co & Mo & Cr & Fe & Ni \\
\hline Q235 & 0.3 & 0.15 & $/$ & $/$ & $/$ & $/$ & Bal. & $/$ \\
HX & 0.247 & 0.264 & 0.201 & 0.875 & 9.16 & 22.34 & 18.15 & Bal. \\
\hline
\end{tabular}

A Trans Tig5000 Series Digital automatic welding machine (Fronius, Pettenbach, Austria) was adopted to fabricate the coatings. Figure 1 shows a schematic diagram of plasma-arc surfacing welding. First, the non-arc power supply causes the non-arc between the tungsten electrode and the nozzle. Once the non-arc is produced, the plasma gas between the tungsten electrode and the nozzle causes the formation of the path. When the main arc power supply is turned on, the arc will start between the base metal and the tungsten electrode. The main arc is then generated, also known as the transfer arc. Because the current of the main arc is generally large, the energy density of the main arc is also relatively high, which can form a molten pool on the surface of the base metal. Welding wire enters the plasma flame, is melted by the plasma flame, and is deposited on the base metal to form a coating. Protective gas protects the plasma flame from oxidation of the molten pool by oxygen during surfacing welding through a protective gas channel. The direction of the arc movement is the direction of deposition. Due to the influence of dilution, 
different surfacing layers may affect the performance of the coatings. Two coatings were deposited by HX wire: one was HX1 coating with one layer of HX; the other was HX2 coating with two layers of HX. Table 2 shows the surfacing welding process parameters. Argon, with a purity of $99.99 \%$, was used as a protective gas throughout these processes.

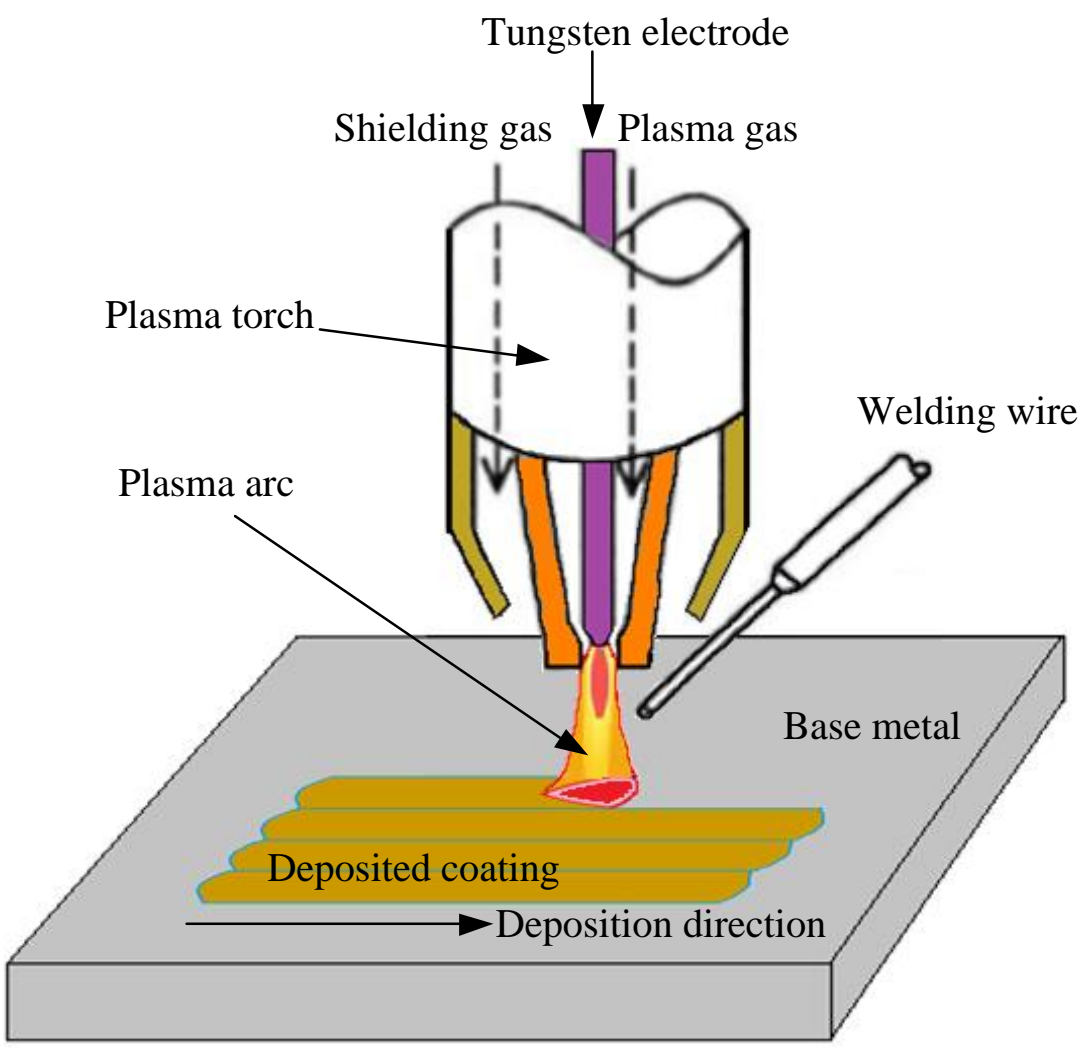

Figure 1. A schematic diagram of plasma-arc surfacing welding.

Table 2. The parameters of the plasma-arc welding process.

\begin{tabular}{cccccc}
\hline Parameters & $\begin{array}{c}\text { Welding Voltage } \\
\text { (V) }\end{array}$ & $\begin{array}{c}\text { Welding Current } \\
\text { (A) }\end{array}$ & $\begin{array}{c}\text { Welding Speed } \\
\text { (cm/min) }\end{array}$ & $\begin{array}{c}\text { Welding Torch } \\
\text { Height (mm) }\end{array}$ & $\begin{array}{c}\text { The Plasma Gas } \\
\text { Flow (L/min) }\end{array}$ \\
\hline Value & 20.1 & 120 & 24 & 7 & 2.5 \\
\hline
\end{tabular}

\subsection{Microstructure Observation and Hardness Measurement}

Square coatings with dimensions of $10 \times 10 \mathrm{~mm}^{2}$ were cut and polished for the XRD test, microstructure observation, and hardness measurement.

The main chemical compositions of the HX1 and HX2 coatings were tested by a direct-reading spectrograph with a PAD5500II (Shimadzu, Kyoto, Japan). The crystal structures of $\mathrm{CoCrFeNiSiMoW}$ coatings were tested by X-ray diffractometer (XRD, Bruker

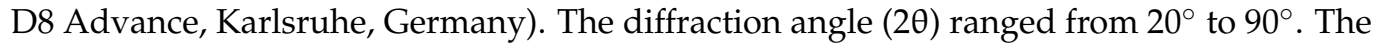
scan rate was $5^{\circ}$ per minute with a step size of $0.02^{\circ}$. The microstructures of the coatings were characterized by an optical microscope (Nikon Epiphot 300 model, Tokyo, Japan) and a scanning electron microscope (SU-70 model, Tokyo, Japan) after the coatings were mechanically polished and etched in a $3 \mathrm{wt} \% \mathrm{HNO}_{3}$ solution.

A DHV-1000 hardness tester (Shanghai Shangcai Tester Machine Co., LTD., Shanghai, China) was used to measure the surface hardness of the HX1 and HX2 coatings every 0.25 $\mathrm{mm}$ with a test load of $500 \mathrm{~g}$ and a dwell time of $15 \mathrm{~s}$. These experiments were repeated five times. The test result was the average value of five experiments. The ranges between 
plus and minus were obtained by calculating the standard deviation of five experimental data. The horizontal axis was the distance, and the vertical axis was the hardness value.

\subsection{Wear Measurements}

Square coatings with dimensions of $30 \times 30 \mathrm{~mm}^{2}$ were cut and polished for a wear resistance test. A dry reciprocating sliding wear test was carried out by the HSR-2M friction tester (Lanzhou Institute of Chemical Physics, Lanzhou, China) at room temperature. A $\mathrm{Si}_{3} \mathrm{~N}_{4}$ ceramic ball was used as the friction couple, and the working disk was coated in $\mathrm{HX} 1$ and $\mathrm{HX} 2$. The $\mathrm{Si}_{3} \mathrm{~N}_{4}$ ball was reciprocated with a stroke length of $5 \mathrm{~mm}$ at a sliding speed of $25 \mathrm{~mm} / \mathrm{s}$ for a duration of $30 \mathrm{~min}$. The loads were chosen to be 20, 30, and 40 N. During the friction testing, the data were transmitted to a computer by the sensor on the loading rod, and the corresponding friction coefficient was calculated. The friction tests were repeated at least five times. The test results are the average of five experiments. After the friction tests, images of wear scars were observed by a laser scanning confocal microscope (LEXT, OLS400 LSCM, Tokyo, Japan) and a scanning electron microscope (SEM, JEOL, JSM-6480, Tokyo, Japan). The wear rates $\left(\mathrm{mm}^{3} \mathrm{~N}^{-1} \cdot \mathrm{m}^{-1}\right)$, represented by $W_{S}$, can be calculated by the following formula [22]:

$$
W_{S}=\frac{C A}{F L}
$$

where parameters $C, A, F$ and $L$, correspond to the width of the wear scar (mm), average wear area of wear loss $\left(\mathrm{mm}^{2}\right)$, applied load $(\mathrm{N})$, and the distance of sliding friction $(\mathrm{mm})$, respectively.

\subsection{Electrochemical Corrosion}

An electrochemical corrosion analysis of $\mathrm{CoCrFeNiSiMoW}$ coatings was conducted in 3.5\% $\mathrm{NaCl}$ solution by a CHI600E electrochemical workstation (Wuhan Corrtest Instruments, Corp., Wuhan, China) at room temperature. A three-electrode electrochemical cell, including a saturated calomel reference electrode (SCE), a platinum counter electrode, and a working electrode of coatings of HX1 and HX2, was adopted [23]. The scan rate of the tests was $0.5 \mathrm{mV} / \mathrm{s}$, and the test range was $-0.5 \sim 1.5 \mathrm{~V}$.

\section{Results and Discussion}

\subsection{Morphology Observation}

The surface appearance of CoCrFeNiSiMoW coatings after plasma-arc surfacing welding is shown in Figure 2. It is evident that the HX wires were melted sufficiently, and continuous coatings were formed on the Q235 base metal. Each coating had a uniform geometric appearance, and the surface of the HX2 coating was smoother than that of HX1 coating. The overlaps between adjacent welding passes were almost parallel to each other.

Figure 3 shows the optical microstructures of the HX1 and HX2 coatings. From Figure $3 a$, it can be seen that the microstructure of the HX1 coating contains various forms of equiaxed microstructures, epitaxial dendrites, and cellular crystals. Interface also can be seen in the overlap area between welding passes, which can be attributed to different growth velocities and different growth directions. Figure $3 \mathrm{~b}$ shows a typical equiaxed microstructure on the surface of the HX2 coating. Figure $3 c-e$ is the microstructure of the cross section of the HX2 coating: the bottom, medium and top, respectively. It can be seen that from the bottom to the top, i.e., the deposition direction, dendrites grew in different directions. There are tiny sedimentary facies between dendrites. These smaller deposits are evenly distributed between dendrites at the bottom and middle. At the top, the dendrites are coarse, and there are more small precipitates of particles between the dendrites. This is due to the sectioning of the epitaxial dendrites growing upward along the deposition direction. Compared with the HX1 coating shown in Figure 3a, the microstructure of the HX2 coating shown in Figure $3 b$ is obviously smaller than that of the HX1 coating, which may lead to the higher hardness of the HX2 coating. 


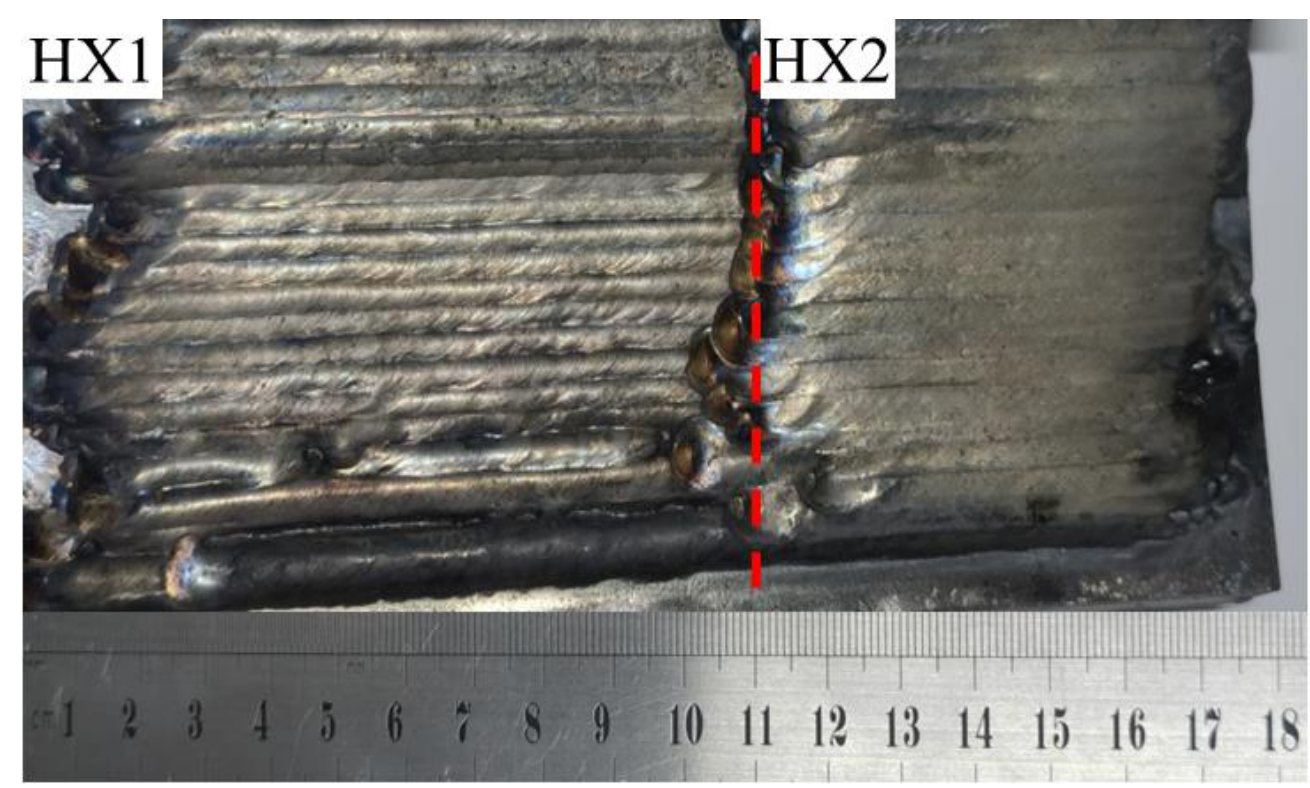

Figure 2. The surface appearance of $\mathrm{CoCrFeNiSiMoW}$ coating after plasma-arc surfacing welding. $\mathrm{HX} 1$ is a one-layer coating, and HX2 is a two-layer coating.
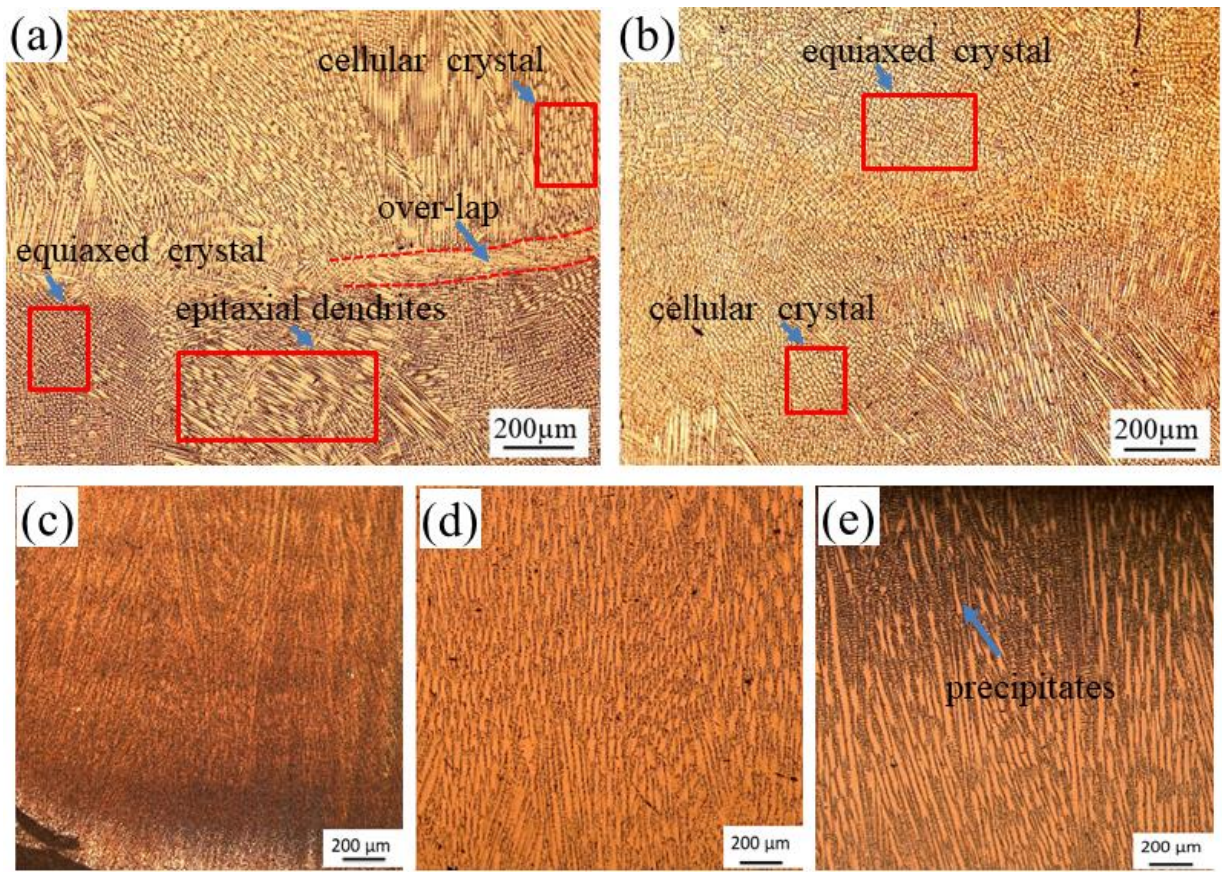

Figure 3. Optical microstructures of the HX1 and HX2 coatings: (a) the microstructure of the surface of the HX1 coating; (b) the microstructure of the surface of HX2 coating; (c-e) the microstructures of the bottom, medium, and top cross-sections of the HX2 coating, respectively.

\subsection{XRD Analysis}

Table 3 shows the main elemental compositions of the HX1 and HX2 coatings. It was shown that they were the CoCrFeNiSiMoW coatings. The entropy value $(\Delta S)$ of the coatings can be obtained by Boltzmann's hypothesis [24]:

$$
\Delta S=-R\left[X_{1} \ln X_{1}+X_{2} \ln X_{2}+\ldots X_{n} \ln X_{n}\right]=-R \sum_{i=1}^{n} X_{i} \ln X_{i}
$$


where $R$ is a gas constant, $X_{i}$ is the molar ratio of the principal element, and $n$ is the number of mixing elements. According to the value of $\Delta S$, alloys are divided into high-, medium-, and low-entropy alloys. When the value of $\Delta S$ is greater than or equal to $1.5 R$, it is considered to be a high-entropy alloy. When the value of $\Delta S$ is between $R$ and $1.5 R$, it is considered to be a medium-entropy alloy. If the value of $\Delta S$ is less than $R$, it is identified as a low-entropy alloy [24].

According to Formula (2), the mixing entropy of the alloying layers' system can be calculated by the molar fraction of each element. The mixed entropy of the HX1 and HX2 coatings was $1.30 R$ and $1.28 R$, respectively. Therefore, the HX1 and HX2 coatings can be considered CoCrFeNiSiMoW medium-entropy coatings.

Table 3. Analysis results of the elemental composition on the surface of the HX1 and HX2 coatings (mole fraction, \%).

\begin{tabular}{cccccccc}
\hline Elements & Ni & Cr & Fe & Mo & Co & W & Si \\
\hline Coating HX1 & 42.21 & 18.85 & 31.85 & 4.43 & 1.28 & 0.15 & 1.23 \\
Coating HX2 & 44.37 & 19.95 & 28.53 & 4.81 & 2.02 & 0.16 & 0.90 \\
\hline
\end{tabular}

From Table 3, it can be seen that the Ni, Cr, Co, W, and Mo contents in the HX2 coating are higher than in the HX1 coating. Ni, Cr, and Co can be formed in solid solution. The elements of W and Mo can solubilize in solid solution, which contributes to solid solution strengthening. The atomic radii of Mo and $\mathrm{W}$ are larger than that of the other elements involved, and the solid solubility of the W and Mo in the Ni-Cr-Co solid solution matrix is large. They can cause lattice deformations, resulting in a solid solution, as has been reported previously $[25,26]$. This may lead to the higher hardness of the HX2 coating compared to the HX1 coating.

The XRD patterns of the HX1 and HX2 coatings are shown in Figure 4 . It can be confirmed that the CoCrFeNiSiMoW coatings of HX1 and HX2 display a solution matrix of CoCrFeNiSiMoW cubic phase (FCC), according to JCPDS card number 35-1489. The high mixing entropy effect can effectively reduce the mixing Gibbs free energy, thus promoting the formation of a FCC solid solution during solidification [14]. In addition to the FCC matrix, some weak diffraction peaks were detected. These diffraction peaks correspond to some secondary phases. Their structures are similar to (Fe, Ni), Mo1.24Ni0.76, and CoCx phases, according to JCPDS card numbers 47-1417, 47-1129, and 44-0962, respectively. Compared with the XRD diffraction peak of HX, the peak of the HX2 coating is shifted to the left. The stronger lattice distortion is caused by the higher content of $W$ and Mo in the HX2 coating [27].

\subsection{Hardness of Coatings}

Figure 5 shows the surface hardness profile of the HX1 and HX2 coatings. The hardness values of the HX1 and HX2 coatings fluctuate from 165 to $195 \mathrm{HV}$ and from 192 to $200 \mathrm{HV}$, with average hardness values of $186 \pm 1.56$ and $198 \pm 1.78 \mathrm{HV}$, respectively. The hardness of the HX2 coating is higher than that of the HX1 coating. The hardness tests are consistent with the analysis results in Figure 3 and Table 3. Hardness represents the ability of materials to resist residual deformation and anti-failure. In general, the higher the hardness of the material, the better the wear resistance. 


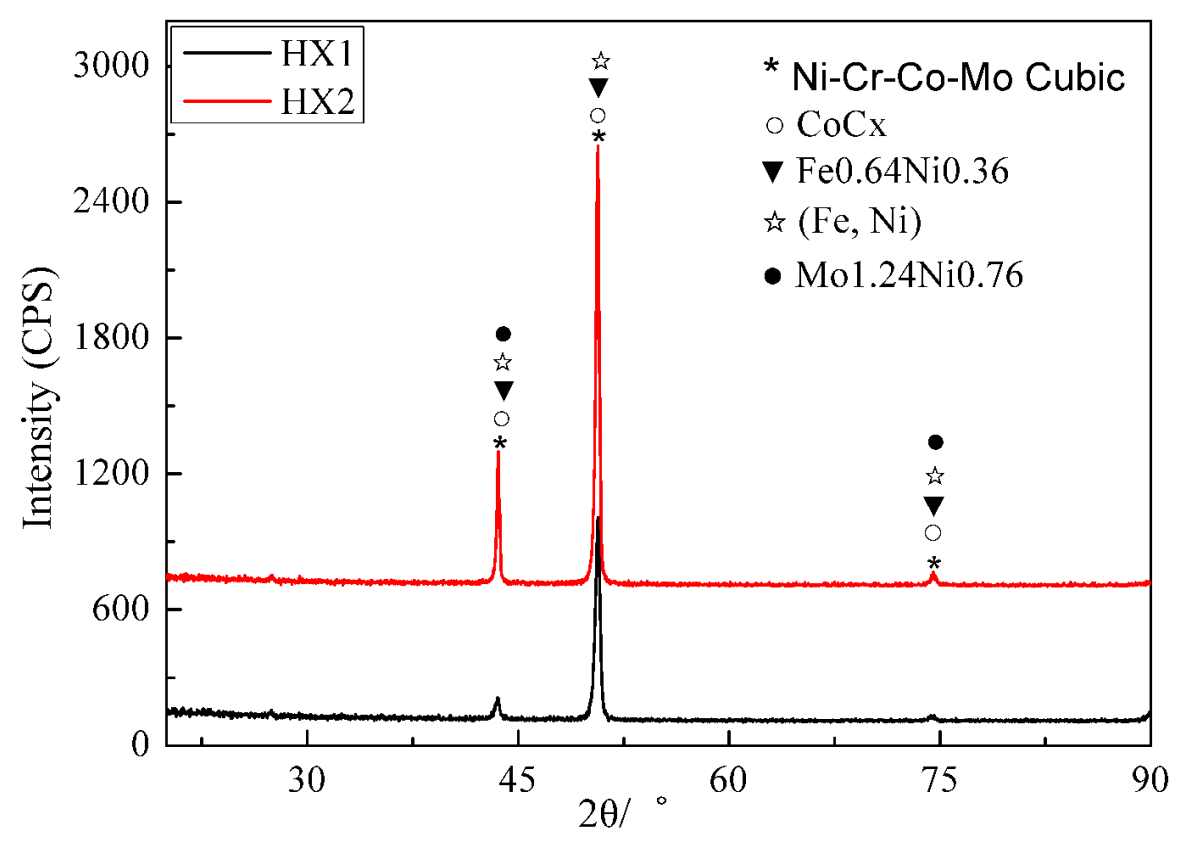

Figure 4. XRD patterns of the HX1 and HX2 coatings.

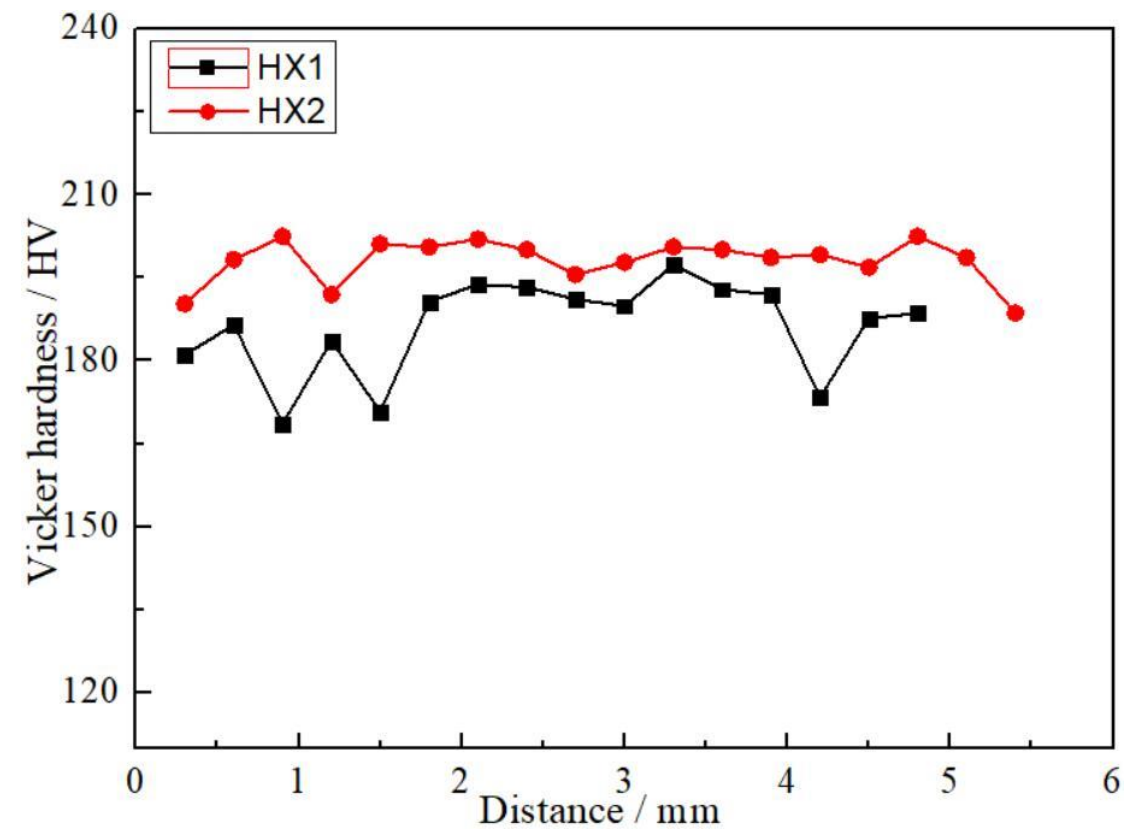

Figure 5. The surface hardness of the $\mathrm{HX} 1$ and $\mathrm{HX} 2$ coatings.

\subsection{Wear Analysis}

Figure 6 represents the change in the friction coefficients of CoCrFeNiSiMoW MEA coatings as a function of wear test time under different loads. During test periods of $150 \mathrm{~s}$, the friction coefficients increased over time, then declined and remained fluctuating. The increase indicates the transition from material intact to material fracture [28]. It also can be seen that the friction coefficient decreased with the increased load. Under the same load, the friction coefficient of the HX2 coating was less than that of the HX1 coating. It indicates that the change of the friction coefficient might relate to the wear mechanism caused by the changing load. The wear mechanism will be further analyzed later in detail. 

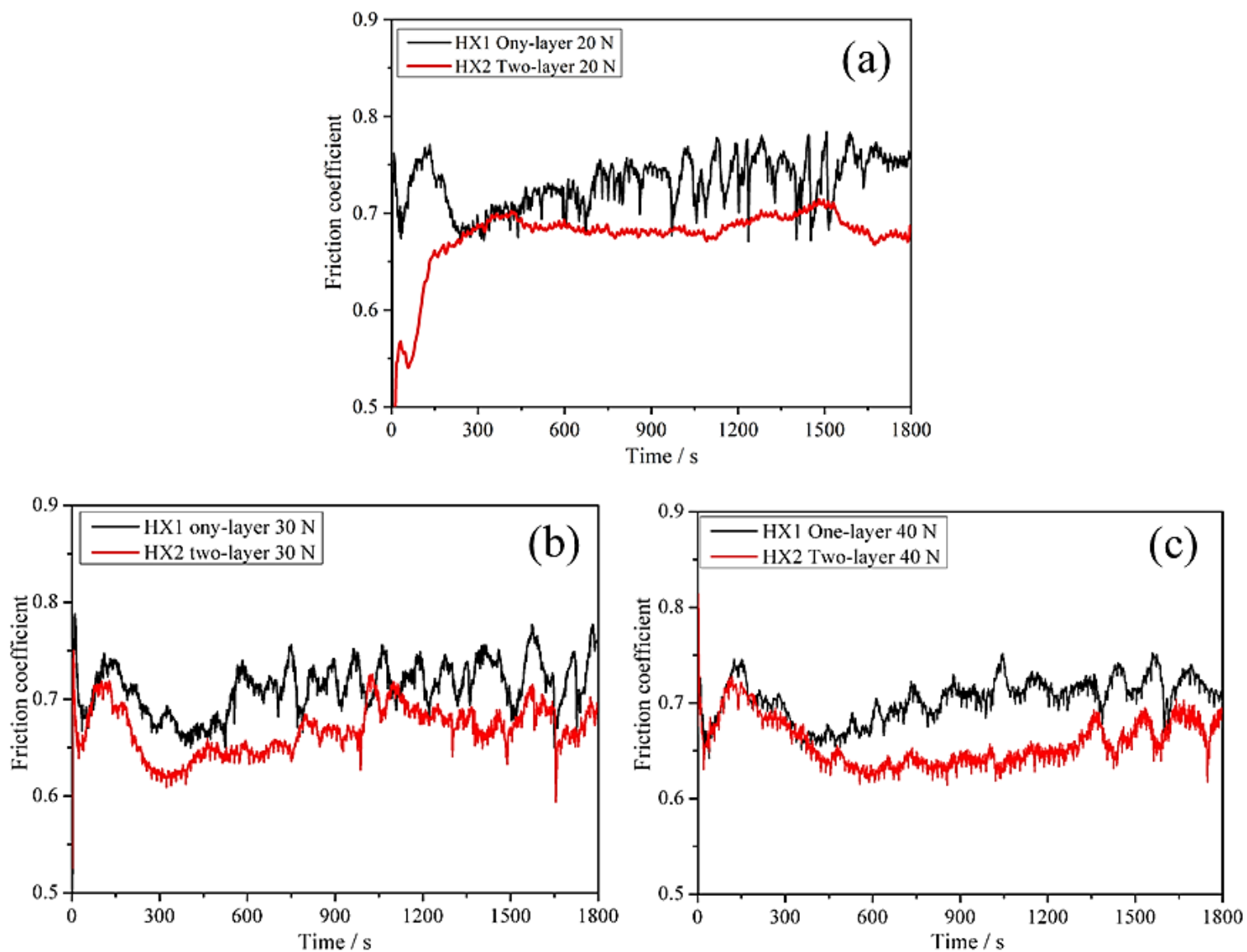

Figure 6. The change of friction coefficient of the HX1 and HX2 coatings as a function of time under different loads. $20 \mathrm{~N}$ (a), $30 \mathrm{~N}(\mathbf{b}), 40 \mathrm{~N}(\mathbf{c})$.

Figure 7 represents the wear rate of the HX1 and HX2 coatings with increasing loads. The wear rate of the HX1 coating increased from 0.348 to $0.489 \mathrm{~mm}^{3} \cdot \mathrm{N}^{-1} \cdot \mathrm{m}^{-1}$, while the wear rate of the HX2 coating increased from 0.341 to $0.439 \mathrm{~mm}^{3} \cdot \mathrm{N}^{-1} \cdot \mathrm{m}^{-1}$ when the loads increased from 20 to $40 \mathrm{~N}$. This demonstrates that the wear rate increased with increasing loads. Comparing the HX1 and HX2 coatings at a load of $20 \mathrm{~N}$, the wear rates of HX1 and HX2 are 0.348 and $0.341 \mathrm{~mm}^{3} \cdot \mathrm{N}^{-1} \cdot \mathrm{m}^{-1}$, respectively, which is a statistically insignificant difference. This means that the performance of both coatings is similar at a load of $20 \mathrm{~N}$. At a load of 30 or $40 \mathrm{~N}$, the wear rate of HX2 is lower than that of HX1. Wear belongs to the category of contact surface stress, that is, the wear rate under pressure due to the interaction between the surface of the coating and the $\mathrm{Si}_{3} \mathrm{~N}_{4}$ ball. In this case, the wear resistance of a high hardness coating is better than that of low hardness coating. The test results are consistent with the analysis of the hardness test results.

Figure 8 presents the wear scars of the HX1 and HX2 coatings under different loads. When the applied loads change from 20 to $40 \mathrm{~N}$, it can be seen that the widths of the wear scars of the HX1 and HX2 coatings increase from 1007.60 to $1264.86 \mu \mathrm{m}$, and from 982.10 to $1192.85 \mu \mathrm{m}$, respectively. The width of the wear scars increases with the increase in applied load. In Figure $8 \mathrm{a}-\mathrm{c}$, there is a visibly large quantity of continuous spalling surfaces and some ploughs on the worn surface of the HX1 coating. It indicates that the main wear mechanism of the HX1 coating is adhesion wear, followed by abrasive wear. For the HX2 coating, as shown in Figure $8 \mathrm{~d}-\mathrm{f}$, there is a large number of grooves and some discontinuous spalling surfaces. It means that the main wear mechanism of the HX2 coating is abrasive wear, followed by adhesion wear. 


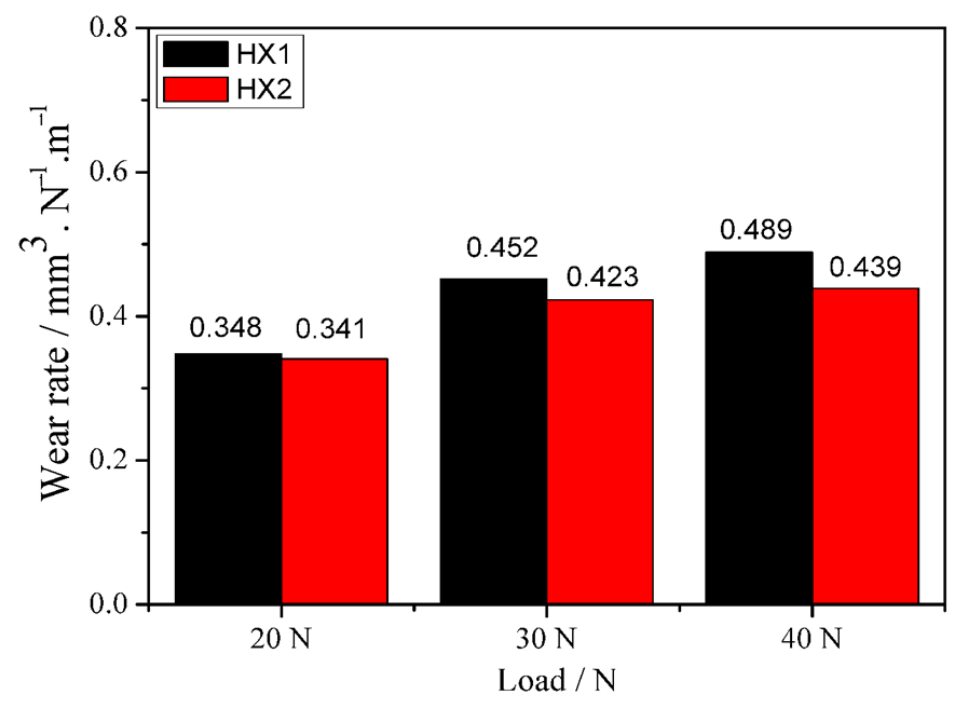

Figure 7. Wear rate of the HX1 and HX2 coatings under different applied loads.
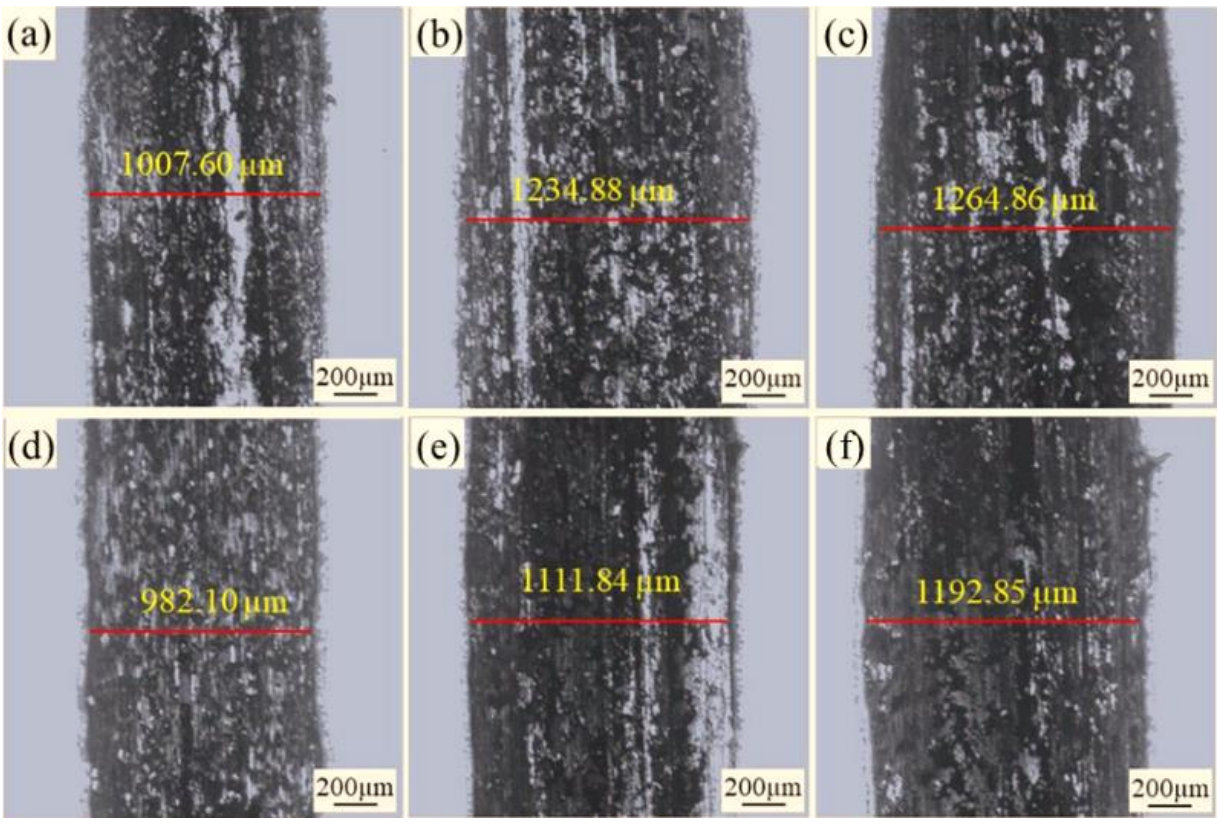

Figure 8. LSCM images of the wear scars under different loads: $20 \mathrm{~N}(\mathbf{a}, \mathbf{d}) ; 30 \mathrm{~N}(\mathbf{b}, \mathbf{e}) ; 40 \mathrm{~N}(\mathbf{c}, \mathbf{f})$. $(\mathbf{a}-\mathbf{c})$ The images of the HX1 coating; $(\mathbf{d}-\mathbf{f})$ the images of the HX2 coating.

Figure 9 plots the two-dimensional (2D) profiles of the cross-section of the wear scars under different loads. When the load increased from 20 to $40 \mathrm{~N}$, the depths of the wear scars on the HX1 and HX2 coatings increased from 11.5 to $21.5 \mu \mathrm{m}$, and from 10.5 to $20.8 \mu \mathrm{m}$, respectively. The width of the wear scar also increased with the increasing load. The wear track depth of the HX1 coating was slightly larger than that of the HX2 coating after sliding wear under the same load. The edges of the HX1 and HX2 coatings became evident due to extrusion with the load increases.

Figure 10a-f shows the worn surface morphology of the HX1 and HX2 coatings examined in detail using SEM after being subjected to sliding wear under different loads. From Figure 10a, it can be seen there were flake debris, spalling from the surface of the HX1 coating, and broken debris under a load of $20 \mathrm{~N}$. When the load increased to $30 \mathrm{~N}$, flake debris, spalling, and broken debris were aggravated, and cracks appeared, as shown in Figure 10b. When the load reached $40 \mathrm{~N}$, the flake debris and surface appalling shown in Figure 10c changed little compared with that shown in Figure 10b. This morphology is consistent with the analysis results in Figure 8a-c. The main wear mechanism of the HX1 
coating is adhesion wear, followed by abrasive wear. From Figure 10d, it can be seen there were wear debris and a discontinuous glaze layer on the worn surface of the HX2 coating. Cracks and broken debris appeared, and wear debris was visible on the surface of the HX2 coating, as shown in Figure 10e,f. It indicates that the wear mechanisms of the HX2 coating is adhesion and abrasive wear. The significant fluctuations in the friction coefficients, as shown in Figure 6, are attributable to inhomogeneous distributions of the glaze layer and wear debris.

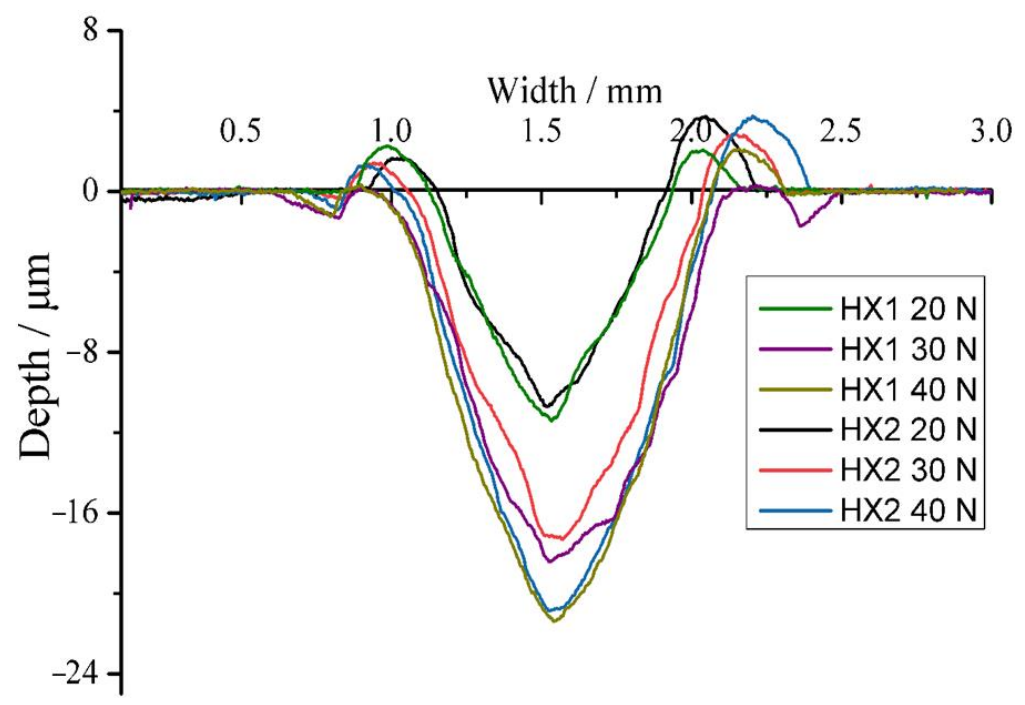

Figure 9. Two-dimensional (2D) profiles of the cross-section of the wear scars under different loads.
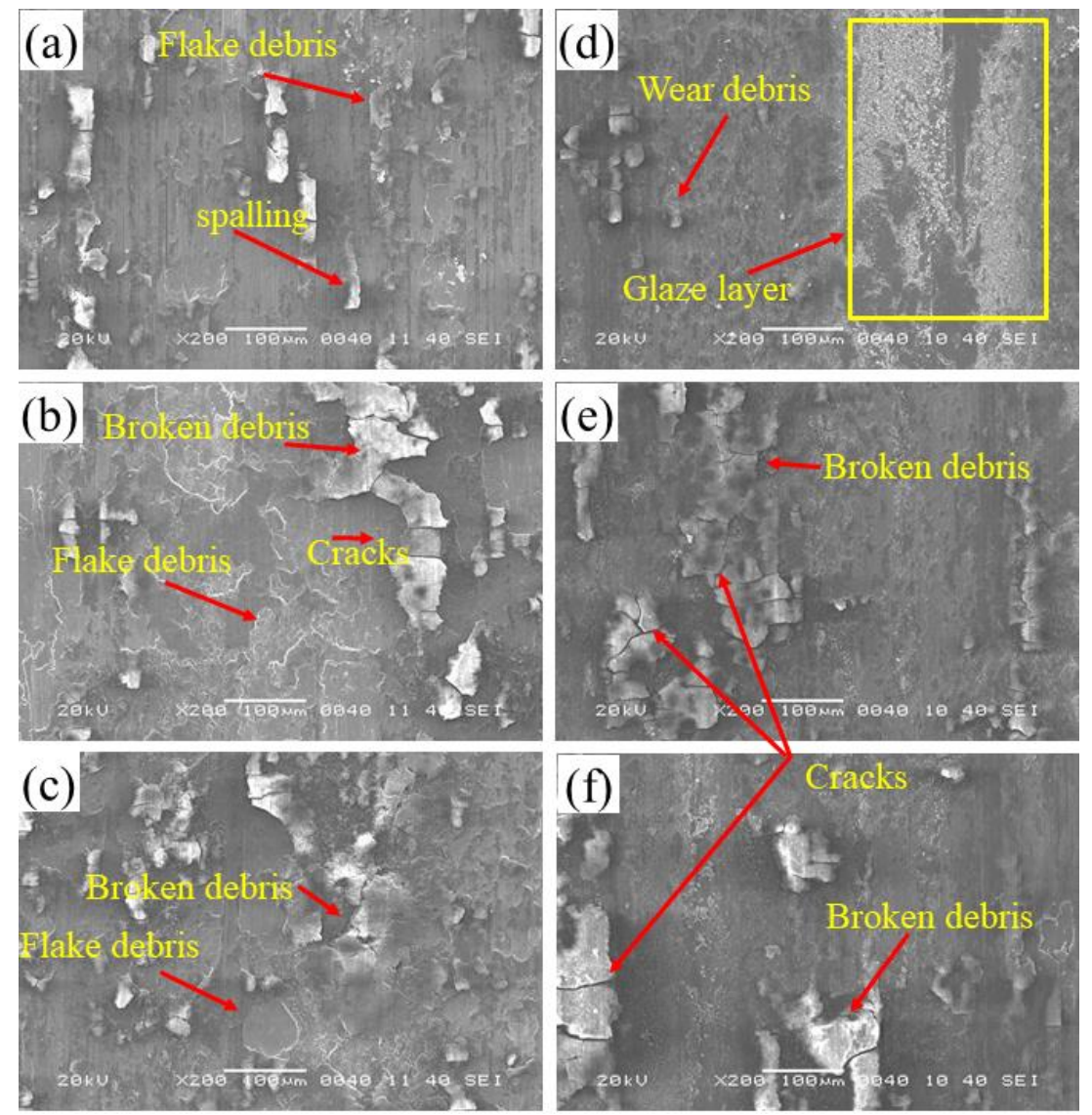

Figure 10. SEM micrographs of the worn surface under different loads: $20 \mathrm{~N}(\mathbf{a}, \mathbf{d}) ; 30 \mathrm{~N}$ (b,e); $40 \mathrm{~N}$ $(\mathbf{c}, \mathbf{f})$. $(\mathbf{a}-\mathbf{c})$ Images of the HX1 coating; $(\mathbf{d}-\mathbf{f})$ images of the HX2 coating. 


\subsection{Electrochemical Corrosion}

Figure 11 presents the polarization curve of the CoCrFeNiSiMoW MEACs in a 3.5\% $\mathrm{NaCl}$ solution. Both MEACs underwent passivation, even though the two MEACs exhibit the same corrosion potential. The corrosion current density of the two coatings increased rapidly when the potential was about $1.0 \mathrm{~V}$ and passive corrosion occurred, which can obstruct the corrosion of the coating surface by the $3.5 \% \mathrm{NaCl}$ solution.

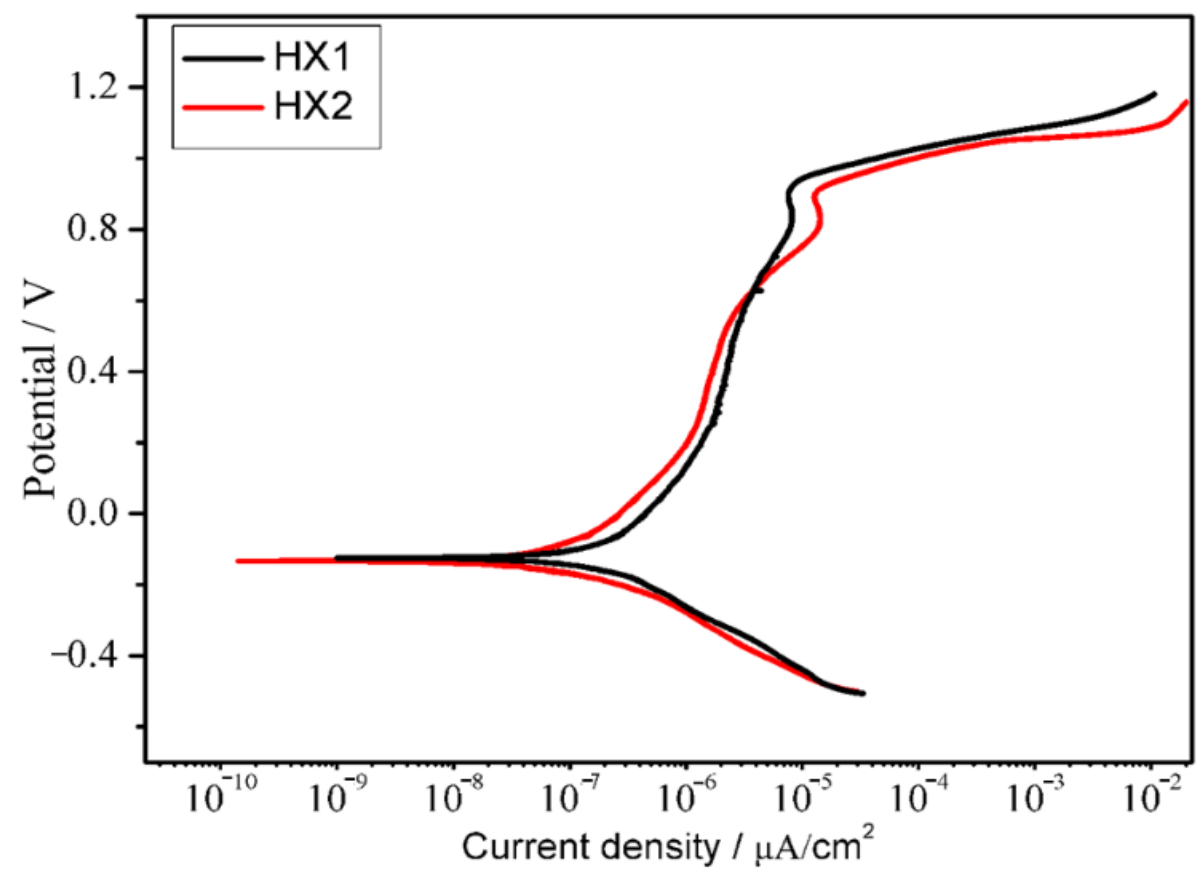

Figure 11. Polarization curve of the CoCrFeNiSiMoW MEACs in a $3.5 \% \mathrm{NaCl}$ solution.

Table 4 shows the free corrosion potential (Ecorr) and the current density (icorr) value, which are determined from the linear portion of the polarization curve by Tafel extrapolation. According to classical electrochemical theory, the free corrosion potential (Ecorr) only represents the thermodynamic trend in the coatings in the corrosion process, while the free corrosion density (Icorr) is caused by the dissolution of the coatings. Therefore, Icorr is more accurate for evaluating corrosion resistance of coatings [29,30]. Compared to the HX1 coating, the HX2 coating has a lower Ecorr and smaller Icorr. It can be concluded that the HX2 coating has better corrosion resistance than the HX1 coating.

Table 4. Corrosion potential and corrosion current density of the coatings in a $3.5 \% \mathrm{NaCl}$ solution.

\begin{tabular}{ccc}
\hline Coating & Ecorr (VSCE) & Icorr $\left(\boldsymbol{\mu} \mathbf{A} / \mathbf{c m}^{2}\right)$ \\
\hline HX1 & -0.12468 & $4.0998 \times 10^{-7}$ \\
HX2 & -0.13313 & $9.3562 \times 10^{-8}$ \\
\hline
\end{tabular}

Figure 12 shows SEM images of corrosion of the two HX coatings. In Figure 12a, it can be seen that the dendrite core of the HX1 coating and the deposited particles between cellular crystals were corroded, while the dendrite core of the HX2 coating remained intact, and the deposited particles between dendrites were visible, as shown in Figure 12b. On the one hand, this result can be attributed to some large dendrites on the surface of the HX1 coating, as shown in Figure 3a, and the finding that the boundaries between the dendrites maximized the interaction area between the surface of the HX1 coating and the corrosive solution. On the other hand, it can be seen in Table 3 that the HX2 coating contained a higher amount of Ni than the HX1 coating, and Ni has a higher electrochemical potential, 
which is also beneficial to improving the corrosion resistance of the HX2 coating. The SEM observation results are consistent with the analysis in Figure 11.
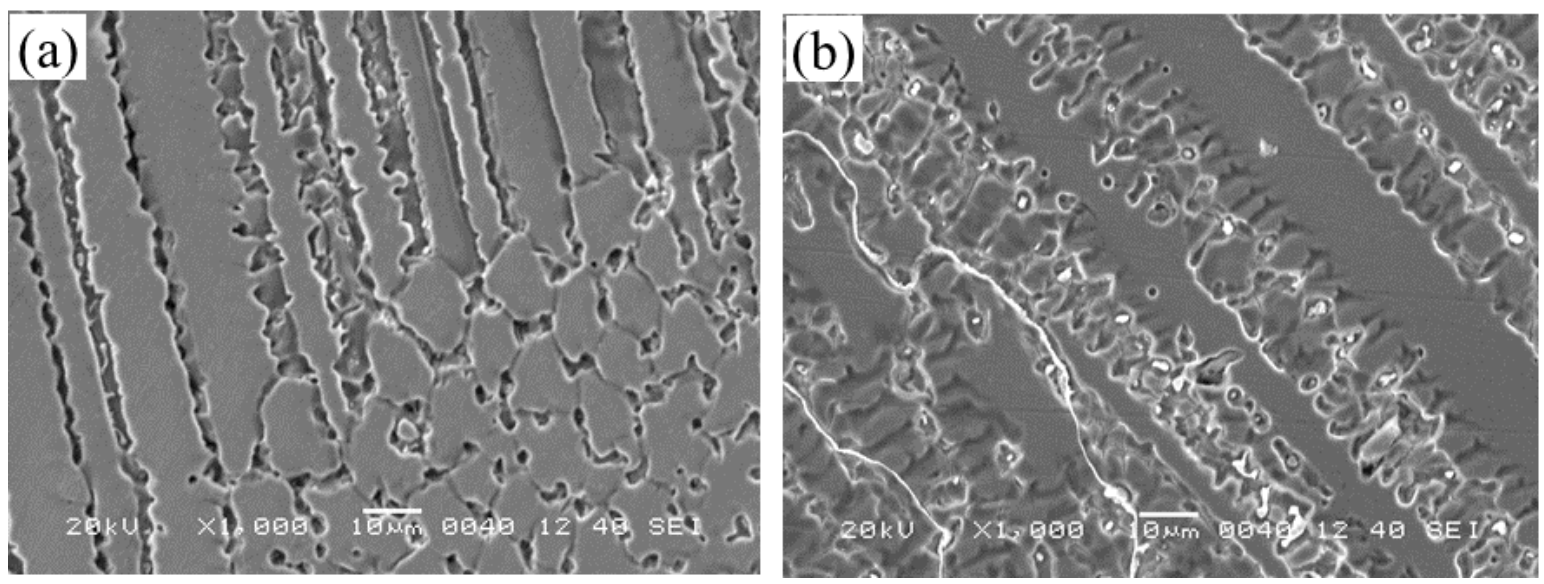

Figure 12. SEM images of corrosion: (a) image of the one-layer HX1 coating; (b) image of the two- layer HX2 coating.

\section{Conclusions}

The CoCrFeNiSiMoW medium-entropy alloy coating was successfully fabricated by plasma-arc surfacing welding.

The CoCrFeNiSiMoW MEACs are a mixture of a NiCrCoMo cubic (FCC) solid solution phase, (Fe, Ni), Mo1.24Ni0.76, and CoCx phases.

The average hardness values of the one-layer and two-layer CoCrFeNiSiMoW MEACs are $186 \pm 1.56$ and $198 \pm 1.78 \mathrm{HV}$, respectively. The two-layer coating has a higher hardness because of a higher content of $\mathrm{W}$ and Mo on the surface of the coating. The radii of $\mathrm{W}$ and Mo atoms are larger than those of the other elements in the coating, and they solubilize in the Ni-Cr-Co solid solution and cause lattice deformation, resulting in solid solution strengthening.

At a load of $20 \mathrm{~N}$, the one-lay coating and two-layer coatings have similar wear resistance. At loads of 30 and $40 \mathrm{~N}$, the wear resistance of the two-layer coating is better than that of a one-layer coating because of the higher hardness of the two-layer coating.

The corrosion resistance of the two-layer CoCrFeNiSiMoW coating is better than that of a one-layer coating because the two-layer coating contains a higher amount of $\mathrm{Ni}$ than a one-layer coating, and $\mathrm{Ni}$ has a higher electrochemical potential, which is also beneficial to improving the corrosion resistance, which is evident in the two-layer coating.

Author Contributions: Project administration, Q.H.; data curation, X.W.; formal analysis, X.S., F.F. and Z.T. All authors have read and agreed to the published version of the manuscript.

Funding: The National Science Foundation of China (Nos. 51205176 and 51675249) supported this research.

Institutional Review Board Statement: Not applicable.

Informed Consent Statement: Not applicable.

Data Availability Statement: The data used to support the findings of this study are available from the corresponding authors upon request.

Conflicts of Interest: The authors declare no conflict of interest. 


\section{References}

1. Zhang, Z.T.; Axinte, E.; Ge, W.J.; Shang, C.Y.; Wang, Y. Microstructure, mechanical properties and corrosion resistance of CuZrY/Al, Ti, Hf series high-entropy alloys. Mater. Des. 2016, 108, 106-113. [CrossRef]

2. Wu, P.H.; Peng, Z.; Liu, N.; Niu, M.Y.; Zhu, Z.X.; Wang, X.J. The effect of Mn content on the microstructure and properties of CoCrCu0.1Fe0.15Mo1.5MnxNi. Mater. Trans. 2016, 57, 5-8. [CrossRef]

3. Chuang, M.H.; Tsai, M.H.; Wang, W.R.; Lin, S.J.; Yeh, J.W. Microstructure and wear behavior of AlxCo1.5CrFeNi1.5Tiy highentropy alloys. Acta Mater. 2011, 59, 6308-6317. [CrossRef]

4. Ye, Q.F.; Feng, K.; Li, Z.G.; Lu, F.G.; Li, R.F.; Huang, J.; Wu, Y.X. Microstructure and corrosion properties of CrMnFeCoNi high entropy alloy coating. Appl. Surf. Sci. 2017, 396, 1420-1426. [CrossRef]

5. Nair, R.B.; Arora, H.S.; Mukherjee, S.; Singh, S.; Singh, H.; Grewal, H.S. Exceptionally high cavitation erosion and corrosion resistance of a high entropy alloy. Ultrason. Sonochem. 2018, 41, 252-260. [CrossRef] [PubMed]

6. Li, Z.M.; Pradeep, K.G.; Deng, Y.; Raabe, D.; Tasan, C.C. Metastable high-entropy dual-phase alloys overcome the strengthductility trade-off. Nature 2016, 534, 227-230. [CrossRef] [PubMed]

7. Qiu, Y.; Thomas, S.; Gibson, M.A.; Fraser, H.L.; Pohl, K.; Birbilis, N. Microstructure and corrosion properties of the low-density single- phase compositionally complex alloy AlTiVCr. Corros. Sci. 2018, 133, 386-396. [CrossRef]

8. Feng, K.; Zhang, Y.; Li, Z.G.; Yao, C.W.; Yao, L.; Fan, C.Y. Corrosion properties of laser cladded CrCoNi medium entropy alloy coating. Surf. Coat. Technol. 2020, 397, 126004. [CrossRef]

9. Zhao, D.C.; Yamaguchi, T.; Tusbasa, D.J.; Wang, W.Q. Fabrication and friction properties of the AlFeCrCo medium-entropy alloy coating on magnesium alloy. Mater. Des. 2020, 193, 108872. [CrossRef]

10. Tian, Y.; Lu, C.Y.; Shen, Y.F.; Feng, X.M. Microstructure and corrosion property of CrMnFeCoNi high entropy alloy coating on Q235 base metal via mechanical alloying method. Surf. Interfaces. 2019, 15, 135-140. [CrossRef]

11. Wu, W.; Jiang, L.; Jiang, H.; Pan, X.M.; Cao, Z.Q. Phase evolution and properties of Al2CrFeNiMox high-entropy alloys coatings by laser cladding. J. Therm. Spray Technol. 2015, 24, 1333-1340. [CrossRef]

12. Xu, J.; Peng, S.; Li, Z.Y.; Jiang, S.Y.; Xie, Z.H.; Munroe, P.; Lu, H. Remarkable cavitation erosion-corrosion resistance of CoCrFeNiTiMo high-entropy alloy coatings. Corros. Sci. 2021, 190, 109663. [CrossRef]

13. Lloyd, A.C.; Noël, J.J.; Mclntyre, S.; Shoesmith, D.W. Cr, Mo and W alloying additions in Ni and their effect on passivity. Electrochim. Acta 2004, 49, 3015-3027. [CrossRef]

14. Shun, T.T.; Chang, L.Y.; Shiu, M.H. Microstructure and mechanical properties of multiprincipal component CoCrFeNiMox alloys. Mater. Charact. 2012, 70, 63-67. [CrossRef]

15. Hsu, Y.J.; Chiang, W.C.; Wu, J.K. Corrosion behavior of FeCoNiCrCux high-entropy alloys in 3.5\% sodium chloride solution. Mater. Chem. Phys. 2005, 92, 112-117. [CrossRef]

16. Li, Y.Z.; Shi, Y. Microhardness, wear resistance, and corrosion resistance of Al0.8CrFeCoNiCu high-entropy alloy coatings on aluminum by laser cladding. Mater. Res. Express. 2021, 7, 026504.

17. Chen, T.K.; Shun, T.T.; Yeh, J.W.; Wong, M.S. Nanostructured nitride films of multi-element high-entropy alloys by reactive DC sputtering. Surf. Coat. Technol. 2004, 188-189, 193-200. [CrossRef]

18. Zeng, Q.F.; Xu, Y.T. A comparative study on the tribocorrosion behaviors of AlFeCrNiMo high entropy alloy coating and 304 stainless steel. Mater. Today Commun. 2020, 24, 101261. [CrossRef]

19. Wang, F. Mechanical property study on rapid additive layer manufacture Hastelloy $X$ alloy by selective laser melting technology. Int. J. Adv. Manuf. Technol. 2011, 58, 545-551. [CrossRef]

20. Zhao, J.C.; Larsen, M.; Ravikumar, V. Phase precipitation and time temperature-transformation diagram of Hastelloy X. Mater. Sci. Eng. A. 2000, 293, 112-119. [CrossRef]

21. Blue, C.A.; Blue, R.A.; Lin, R.Y.; Lei, J.F.; Williams, W.D. Joining of Hastelloy X to Inconel 718 using an infrared process. J. Mater Process. Technol. 1996, 58, 32-38. [CrossRef]

22. Qiao, Y.X.; Sheng, S.L.; Zhang, L.M.; Chen, J.; Zheng, Z.B. Friction and wear behaviors of a high nitrogen austenitic stainless steel Fe-19Cr-15Mn-0.66N. J. Min. Metall. Sect. B Metall. 2021, 57, 285-293.

23. Qiao, Y.X.; Xu, D.K.; Wang, S.; Ma, Y.J.; Chen, J.; Wang, Y.X.; Zhou, H.L. Effect of hydrogen charging on microstructural evolution and corrosion behavior of Ti-4Al-2V-1Mo-1Fe alloy. J. Mater. Sci. Technol. 2021, 60, 168-176. [CrossRef]

24. Sharma, P.; Dwivedi, V.K.; Dwivedi, S.P. Development of high entropy alloys: A review. Mater. Today Proc. 2021, 43, 502-509. [CrossRef]

25. Liu, N.; Ding, W.; Wang, X.J.; Du, J.J.; Liu, L.X. Microstructure evolution and phase formation of Fe25Ni25CoxMoy Multi-principal component alloys. Metall. Mater. Trans. A 2020, 51, 2990-2997. [CrossRef]

26. Ding, W.; Liu, N.; Fan, J.C.; Cao, J.; Wang, X.J. Diffusion bonding of copper and titanium with an interlayer of CoCrFeMnNi high-entropy alloy. Intermetallics 2021, 129, 107027. [CrossRef]

27. Lin, K.H.; Tseng, C.M.; Chueh, C.C.; Chang, S.Y.; Lo, Y.C.; Wang, C.C.; Lin, S.J.; Yeh, J.W. Different lattice distortion effects on the tensile properties of $\mathrm{Ni}-\mathrm{W}$ dilute solutions and $\mathrm{CrFeNi}$ and $\mathrm{CoCrFeMnNi}$ concentrated solutions. SSRN Electron. J. 2021. [CrossRef] 
28. Xi, Y.T.; Bai, Y.Y.; Gao, K.W.; Pang, X.L.; Yang, H.S.; Yan, L.C.; Volinsky, A.A. Residual stress and microstructure effects on mechanical, tribological and electrical properties of TiN coating on 304 stainless steel. Ceram. Int. 2018, 44, 15851-15858. [CrossRef]

29. Li, Y.Z.; Shi, Y. Microhardness, wear resistance, and corrosion resistance of AlxCrFeCoNiCu high-entropy alloy coatings on aluminum y laser cladding. Opt. Laser Technol. 2021, 134, 106632. [CrossRef]

30. Qiao, Y.X.; Chen, Y.; Li, L.L.; Chen, J.; Emori, W.; Wang, X.J.; Yang, L.; Zhou, H.L.; Song, G.; Naik, N.; et al. Corrosion behavior of a nickel-free high-nitrogen stainless steel with hydrogen charging. JOM 2021, 73, 1165-1172. [CrossRef] 